\title{
ELETROESTIMULAÇÃO EM DOENTES CRÍTICOS: UMA REVISÃO SISTEMÁTICA
}

\author{
Flávio Eduardo Machado da Hora Miranda Fisioterapeuta. Graduado pela Escola Bahiana \\ de Medicina e Saúde Pública. Membro do \\ GEPFIR.
}
Bernardo Costa Alves Dias Fisioterapeuta. Pós-Graduado em Fisioterapia em Terapia Intensiva. Fisioterapeuta do Hospital da Beneficência Portuguesa. Membro do Grupo de Pesquisa em Fisioterapia Cardiovascular e Respiratória da Bahiana (GEPFIR).
Luciana BilitárioMacedo Fisioterapeuta. Docente Escola Bahiana de Medicina e Saúde Pública e Universidade do Estado da Bahia (UNEB). Membro do GEPFIR.

\author{
Cristiane Maria Carvalho Costa Dias Fisioterapeuta. Docente Escola Bahiana de \\ Medicina e Saúde Pública. Fisioterapeuta \\ Líder do Hospital Aliança. Líder do GEPFIR.
}

\begin{abstract}
Resumo
A mobilização precoce é um método viável para a prevenção de problemas físicos e mentais gerados pelo imobilismo, sendo a eletroestimulação neuromuscular (EENM) utilizada como coadjuvante ao exercício ativo e na prevenção da perda da força muscular em pacientes críticos.Objetivo:revelar as evidências científicas sobre os efeitos da EENM em pacientes críticos internados nas unidades de terapia intensiva.Método: trata-se de um estudo de revisão sistemática com ensaios clínicos randomizados, sendo os artigos selecionados na base de dados PUBMED, LILACS e portal CAPES. Os critérios de inclusão foram: abordar sobre a EENM no paciente crítico $\geq 18$ anos de idade, obter escore de qualidade metodológica na Escala de JADAD $\geq 3$, publicados na língua inglesaou portuguesa.As variáveis analisadas foram o perfil clínico, o tempo de aplicação, a quantidade de sessões, os parâmetros utilizados e o desfecho da aplicação da EENM.Conclusão: a aplicação da EENM poderárecuperar músculos enfraquecidos ou retardar o processo de perda muscular, no entanto, os parâmetros publicados encontrados não foram suficientes para sistematizar a técnica.
\end{abstract}

Palavras-chave: Estimulação elétrica; Unidade de terapia intensiva; Doença terminal.

\section{ELECTRICAL ESTIMULATION IN CRITICAL ILLNESS: A SISTEMATIC REVIEW}

\begin{abstract}
Early mobilization is viable method for the prevention of the physical and mental problems generetade by immobilility, and neuromuscularelectrical stimulation(NMES) usedas an adjuncttoexerciseandactivein preventing thelossof muscle strength incritically ill patients. Objective: to clarify the effects os NMES in critical ill patientes hospitalized in intensive care units. Method: this study is a systematic review of randomized clinical trials, and the selected articles in the database PUBMED, LILACS and CAPES. Inclusion criteriawere: approachonNMESin the critically ill patients $\geq 18$ yearsof age,getinmethodologicalquality score $\geq 3$ JADADScale, published in EnglishorPortuguese. The variablesanalyzedwerethe clinical profile, time of application, the number ofsessions, the parameters used and the outcomeof the applicationof NMES. Conclusions: the application of NMESmay actin the recovery ofweakened musclesordelaying the process ofmuscle loss, perhaps the parameters found were not sufficient for systematize the technique.
\end{abstract}

Keywords: Electrical stimulation; Intensive care unit; Criticall illness. 


\section{INTRODUÇÃO}

Em decorrência da crescente e constante evolução tecnológica e científica da medicina intensiva, a sobrevida dos pacientes críticos vem aumentando consideravelmente a cada dia, ${ }^{(1-}$

4) com declínio funcional e consequentemente impacto na qualidade de vida. Em conjunto com o aumento desta sobrevida nestes pacientes, assistimos ao aumento da incidência de complicações decorrentes da permanência prolongada nas Unidades de Terapia Intensiva (UTIs). ${ }^{(1,4,5)}$

A fraqueza muscular adquirida na UTI é uma complicação neuromuscular que acomete entre $30 \%$ a $60 \%$ dos pacientes internados nestas unidades. ${ }^{(6,7)}$ Fatores de risco para o desenvolvimento desta fraqueza incluem: resposta inflamatória sistêmica, o uso de sedativos e bloqueadores neuromusculares, hiperosmolaridade, nutrição parenteral e imobilidade prolongada. ${ }^{(6,8,9)}$

A imobilização e subsequente fraqueza são consequências da doença crítica e podem contribuir para o prolongamento do tempo de hospitalização, declínio no estado funcional e na qualidade de vida que podem persistir mesmo após um ano da alta hospitalar, além de colaborarem para o aumento dos índices de mortalidade e elevação dos custos nas UTIs. ${ }^{(10-12)}$ Estudos recentes tem demonstrado que a mobilização precoce pode ser um meio seguro e viável na prevenção de problemas físicos gerados pelo imobilismo. ${ }^{(6,12)}$ No entanto, a cooperação do paciente é necessária para uma intervenção essencial a ser aplicada. ${ }^{(6)}$

A estimulação elétrica neuromuscular (EENM) tem sido utilizada como alternativa ao exercício ativo e mobilização em pacientes acamados; ${ }^{(6,13,15)}$ a EENM tem mostrados efeitos benéficos em pacientes com doença pulmonar obstrutiva crônica (DPOC), insuficiência cardíaca congestiva (ICC) e em pacientes internados no hospital. ${ }^{(6)}$ Estes pacientes não podem se exercitar ativamente devido a insuficiência respiratória, cardíaca e a sua condição crítica nas UTIs, além da sedação e lesões neurológicas. Por este motivo, se beneficiam da EENM em termos de capacidade de exercício, no desempenho dos músculos esqueléticos, qualidade de vida, redução da perda muscular,prevenção da incidência de polineuromiopatia do paciente crítico,na redução do tempo de desmame e diminuição do tempo de internamento nas UTI. ${ }^{(2,14-17)}$

O perfil clínico dos indivíduos submetidos a sessões de EENM, o tempo de aplicação, o número de aplicações, a quantidade de sessões, os parâmetros utilizados e o desfecho da aplicação da EENM. 
Concomitante com a crescente utilização da eletroestimulação em pacientes críticos,surge a necessidade de fundamentar a sua utilização, realizando umarevisão sistemática. Diante do exposto, este estudo propõe-se a revelar as evidências científicas sobre os efeitos da utilização da EENM em pacientes críticos internados nas UTIs.

\section{MÉTODO}

Trata-se de uma revisão sistemática da literatura. Os artigos científicos para elaboração do mesmo foram selecionados nas bases de dados PUBMED, Biblioteca Virtual em Saúde (BVS) e portal da CAPES nos idiomas inglês e português. Os descritores utilizados foram selecionados nos Descritores em Ciências da Saúde (DeCS): Estimulação Elétrica, Unidade de Terapia Intensiva e Doença Terminal, em português, e ElectricalEstimulation, IntensiveCare Unit e CriticallIllness, em inglês. Para as combinações dos descritores no rastreamento das publicações, o operador “AND” foi utilizado.

Os critérios de inclusão definidos foram: ensaio clínico randomizado, possuírem como critério de inclusão indivíduos $\geq 18$ anos, abordarem sobre a utilização da eletroestimulação no paciente crítico, serem escritos nas línguas inglesa ou portuguesa. A realização da pesquisa constou das seguintes etapas: localização dos estudos pela leitura do título e resumo, leitura criteriosa do resumo dos artigos pré-selecionados, seleção dos artigos coerentes com o objetivo do presente estudo, aplicação da escala de Jadad para avaliação da qualidade metodológica e exclusão dos artigos com escore inferior a três pontos na mesma, interpretação e discussão dos resultados.

Foram identificados inicialmente 872 artigos através da estratégia de busca utilizando os descritores previamente mencionados. Destes, 34 foram selecionados por conterem em seus títulos referência a utilização da eletroestimulação em pacientes internados em unidades de terapia intensiva, sendo selecionados quatro artigos através da leitura crítica dos seus resumos e seleção dos desenhos metodológicos, que deveriam ser do tipo ensaio clínico randomizado (Figura 1). Estes quatro artigos foram submetidos à aplicação da Escala de Jadad, para verificação da qualidade metodológica, onde três estudos obtiveram escore trêse um obteve escore cinco (Tabela 1).

Nos estudos incluídosforam analisados o perfil clínico dos indivíduos submetidos a sessões de EENM, o tempo de aplicação, o número de aplicações, a quantidade de sessões, os parâmetros utilizados e o desfecho da aplicação da EENM. 


\section{RESULTADOS}

Os três estudos analisados tiverem como objetivo avaliar o efeito da eletroestimulação. Entretanto, os perfis dos pacientes avaliados,os parâmetros utilizados e os desfechos avaliadosforam heterogêneos (Tabelas 2 e 3).

Gerovasiliet al. $^{(12)}$ avaliaram a perda de massa muscular em pacientes, Routsi et al. ${ }^{(14)}$ avaliaram o diagnóstico de polineuromiopatia do paciente crítico, ambos analisaram os pacientes à partir do $2^{\circ}$ dia de admissão, com escore APACHE II > 13. Grutheret al. ${ }^{(3)}$ verificaram a capacidade da EENM em retardar a perda da massa muscular e reverter a atrofia muscular em pacientes internados a curto (período entre admissão e início da terapia com EENM inferior a uma semana) e a longo prazo (período entre admissão e início da terapia com EENM superior a duas semanas) nas UTIs. O tamanho das amostras variou de $\mathrm{N}=46^{(3)}$ a $\mathrm{N}=142^{(13)}$ sujeitos.

Quanto a aplicação da corrente, Gerovasiliet al. ${ }^{(12)}$ aplicaram a EENM concomitantemente no quadríceps e no fibular longo nas extremidades inferiores, o tempo de intervenção foi do $2^{\circ}$ ao $9^{\circ}$ dia de internação, com sessões diárias de 55 min. Routsi et al. ${ }^{(14)}$ avaliaram os pacientes durante o período de internação na UTI, com média de 14 dias no grupo intervenção e 22 dias no grupo controle, a aplicação da EENM foi realizada, simultaneamente, nos músculos vasto lateral, vasto medial e fibular longo, as sessões consistiram da aplicação diária durante $55 \mathrm{~min}$ de eletroestimulação até a alta da UTI. Na pesquisa de Grutheret al. ${ }^{(3)}$ a técnica EENM foi aplicada no quadríceps durante um período de quatro semanas, sendo as aplicações realizada uma vez por dia, cinco vezes por semana e o tempo de aplicação de 30 minutos por dia durante a primeira semana e 60 minutos por dia a partir da segunda semana. Esses autores revelaram atraso na diminuição da espessura média da camada muscular de pacientes submetidos a EENM à partir da segunda semana de internamento na UTI.

\section{DISCUSSÃO}


Os estudos avaliados mostraram que a utilização da EENM gera efeitos benéficos sobre o metabolismo muscular. ${ }^{(18)}$ Pacientes criticamente doentes são submetidos a um estado de hipermetabolismo caracterizada por um aumento no gasto de energia, sendo esta condição associada a perda de proteína nos músculos esqueléticos. ${ }^{(19,20)}$ Além disso, a imobilização, mesmo quando de curta duração, leva a degradação da musculatura esquelética. ${ }^{(20,21)}$ Desta forma, a EENM tem sido utilizada como forma alternativa de exercício, gerando um estímulo anabólico que contrapõe os efeitos catabólicos da doença crítica e da imobilização. ${ }^{(13,14)}$

Gerovasiliet al. ${ }^{(12)}$ constataram que a utilização da EENM contribui para a preservação da massa muscular de pacientes criticamente doentes. Routsiet al. ${ }^{(14)}$ observarammelhora da força muscular e diminuição dos dias necessários para o paciente sentar na cadeira nos indivíduos que foram submetidos a sessões diárias de eletroestimulação, assim como, menor tempo na duração da ventilação mecânica e menor tempo de internação nas UTI's. Gruther et al. ${ }^{(3)}$ mostraram atraso na diminuição da espessura média docamada muscular de pacientes submetidos a EENM à partir da segunda semana de internamento na UTI.

Comparativamente, em estudos com animais, foi observado que os níveis de diversas citocinas, principalmente a IL-6, elevam-se após o exercício; também foi observada sua elevação no músculo esquelético em ratos após a aplicação da EENM. Em conjunto com o achado de que a eletroestimulação gera um efeito agudo sistêmico exercido na microcirculação de pacientes criticamente doentes, observou-senos estudos a otimização do tempo de desmame da ventilação mecânica e do tempo de permanência nas UTI's dos pacientes criticamente doentes. ${ }^{(13,22-24)}$

Apesar das escassas pesquisas encontradas sobre os efeitos da eletroestimulação no paciente crítico, esta é utilizada amplamente como alternativa ao exercício ativo em pacientes graves, a EENM resultou em melhora da performance muscular, através da contração voluntária máxima, força e a resistência muscular. ${ }^{(6)}$ Em pacientes com Doença Pulmonar Obstrutiva Crônica (DPOC), restritos ao leito sob ventilação mecânica, a utilização da EENM causou aumento na força muscular e reduziu o número de dias para a transferência da cama para a cadeira. ${ }^{(13,16)}$

Ainda em pacientes com DPOC, foi mostrado que a força muscular de quadríceps é inversamente proporcional a utilização de recursos médicos para a manutenção destes pacientes, ou seja, a perda de força muscular em quadríceps, está diretamente relacionada ao aumento do custo do internamento. Desta forma, a escolha da utilização dos membros 
inferiores para a utilização da eletroestimulação, segundo a literatura, é a escolha mais adequada para a avaliação da perda de massa muscular em pacientes graves. ${ }^{(25)}$

$\mathrm{Na}$ literatura aos parâmetros utilizados nos estudos para EENM nos doentes críticos são variáveis e não há consenso sobre como utilizá-los. Desta forma, para estimular eletricamente as fibras do tipo I em indivíduos saudáveis seriam necessários impulsos com frequência de 20 a $50 \mathrm{~Hz}$ e para o tipo IIa e IIb as frequências utilizadas variam de 50 a 120 Hz. ${ }^{(26)}$ Frequências entre 30 e $80 \mathrm{~Hz}$ parecem ideais para a eletroestimulação neuromuscular e o aumento de força ocorre de maneira similar ao aumento de força gerada através de contrações voluntárias, ou seja, o aumento de força depende do aumento da carga, no caso, da intensidade da corrente. ${ }^{(27)}$ Além disso, as fibras rápidas e lentas possuem diferentes respostas ao estímulo elétrico, sendo assim, as fibras lentas respondem ao tempo de pulso superior a 300ms e as fibras rápidas a tempo de pulso inferior a 300ms. Desta maneira, a escolha da frequência, intensidade e da largura de pulso, respectivamente, nos ensaios clínicos avaliados, estão de acordo com a literatura encontrada. Entretanto os tempo on e off não foram bem estabelecidos na literatura estudada. Foi demonstrado que a contração estimulada contínua do músculo esquelético leva a uma fadiga muscular muito rápida, o que implica na queda da força gerada e que quanto menor a proporção do tempo off no ciclo on-off, maiores fadigas ocorrem durante o período de estimulação, devendo serem utilizados, inicialmente, ciclos com um período relativamente longo de repouso. ${ }^{(28,29)}$ Desta forma, quando o músculo estiver treinado, o tempo "off" pode ser reduzido progressivamente, assim como o tempo "on" aumentado em grande proporção, relativamente ao ciclo de estimulação. ${ }^{(30)}$

Este estudo teve como limitações o reduzido número de ensaios clínicos randomizados com avaliação metodológica adequada que abordaram o tema. A variação dos parâmetros utilizados para a eletroestimulação eos diferentes tempos de aplicação e utilização das intervenções, bem comoa heterogeneidade dos desfechos avaliados comprometem as comparações dos efeitos encontrados entre os autores. Além disso, os artigos relacionados ao tema nem sempre utilizaram descritores encontrados ou registrados nos Descritores em Ciências da Saúde (DeCS) da Biblioteca Virtual de Saúde e do MESH (PUBMED).

As publicações encontradas na literatura evidenciaram que a aplicação da EENM nos membros inferiores pode atuar na recuperação de músculos enfraquecidos ou retardar o processo de perda de massa muscular. Além de, reduzir indiretamente o tempo de internação na UTI e o tempo de VM em pacientes críticos que não estão aptos a realizar exercícios 
ativamente. Entretanto, o perfil dos indivíduos, os parâmetros avaliados, o tempo de aplicação e o período que foi aplicada a eletroestimulação foram divergentes.

Este estudo teve como principais limitações o tipo de metodologia utilizada para excluir os artigos, o não registro do trabalho no sistema PRISMA, o não uso de pelo menos dois avaliadores para escolha dos artigos científicos e durante a revisão foi observada a dificuldade do uso dos unitermos pelos pesquisadores e uma grande variabilidade nos instrumentos utilizados para avaliação dos pacientes e dos desfechos da aplicação da EENM em doentes críticos.

As limitações do estudo apontam para a necessidade da realização de pesquisas sobre a eletroestimulação em pacientes críticos nas UTIs, a fim de sistematizar os parâmetros, o tempo e o período para a aplicação da técnica. Outraspesquisas são necessárias para que seja possível esclarecer sobre as alterações morfológicas geradas nos músculos após a EENM, para mensurar a eficiência da técnica nos diversos perfis clínicos dos pacientes críticos.

\section{REFERÊNCIAS}

1. França EET, Ferrari FR,Fernandes PV,Cavalcanti R,Duarte ACM,Aquim EE, et al. Força tarefa sobre a fisioterapia em pacientes críticos adultos: diretrizes da Associação Brasileira de Fisioterapia Respiratória e Terapia Intensiva (ASSOBRAFIR) e Associação de Medicina Intensiva Brasileira (AMIB). [Internet]. [acesso em2009 Nov 11]. Disponível em: http://www.amib.org.br/pdf/DEFIT.pdf

2. Poulsen JB, Moller K, Jensen CV, Kehlet H, Perner A. Effect of transcutaneous electrical muscle stimulation on muscle volume in patients with septic shock. CriticalCare Medicine. [Internet] 2011 [acesso em2011 mar]; 39(3):456-61. Disponível em: http://www.ncbi.nlm.nih.gov/pubmed/21150583

3. GrutherW, Kainberger F, Fialka-Moser V, Paternostro-Sluga T, Quittan M, Spiss C, et al. Effects of neuromuscular electrical stimulation on muscle layer thickness of knee extensor muscles in intensive care unit patients: a piloty study. JournalRehabilitation Medicine. [Internet] 2011 [acesso em2010 jun]; 42(6):593-7. Disponível em: http://www.ncbi.nlm.nih.gov/pubmed/20549166

4. França EET, Ferrari F, Fernandes P, Cavalcanti R, Duarte ACM, et al. Fisioterapia em pacientes críticos adultos: recomendações do Departamento de Fisioterapia da Associação de Medicina Intensiva Brasileira. Revista Brasileira de Terapia Intensiva. [Internet] 2012 [acesso em2012 fev]; 24(1):6-22. Disponível em: http://www.scielo.br/pdf/rbti/v24n1/03.pdf

5. KaratzanosE, Gerovasili V, Zervakis D, Eli-Sophia T, Apostolou K, et. al. Electrical Muscle Stimulation: An Effective Form of Exercise and Early Mobilization to Preserve 
Muscle Strength in Critically Ill Patients. Critical Care Research and Practice. [Internet] 2012 [acessoem2012 jan] Disponivelem: http://www.hindawi.com/journals/ccrp/2012/432752/

6. Maramattom BV, Wijdicks EF. Acute neuromuscular weakness in the intensive care unit. CriticalCare Medicine. [Internet] 2006 [acesso em2006 nov]; 34(11): 2835-41. Disponível em: http://www.ncbi.nlm.nih.gov/pubmed/16932235

7. Schweickert WJ, Hall J. ICU-acquired weakness. Chest. [Internet] 2007 [acesso em2007 mai] 131(5): 1541-9. Disponível em: http://www.ncbi.nlm.nih.gov/pubmed/17494803

8. Truong AD, Fan E, Brower RG, Needham DM. Bench-to-beside review: mobilizing patients in the intensive care unit -- from pathophysiology to clinical trials. CritCare. [Internet] 2009 [acesso em2009 jul]; 13(4):216. Disponível em: http://www.ncbi.nlm.nih.gov/pubmed/19664166

9. Morris PE, Goad A, Thompson C, Taylor K, Harry B,Passmore L, et al. Early intensive care unit mobility therapy in the treatment of acute respiratory failure. CriticalCare Medicine. [Internet] 2008 [acesso em2008 aug] 36(8): 2238-43. Disponível em: http://www.ncbi.nlm.nih.gov/pubmed/18596631

10. Burtin C, Clerckx B, Robbeets C, Ferdinande P, Langer D, Troosters T, et al. Early exercise in critically ill patients enhances short-term functional recovery. CriticalCare Medicine. [Internet] 2009 [acesso em2009 set] 37(9): 2499-505. Disponível em: http://www.ncbi.nlm.nih.gov/pubmed/19623052

11. Silvia APP, Maynard K, Cruz MR,et. al. Efeitos da fisioterapia motora em pacientes críticos: revisão de literatura. Revista Brasileira de Terapia Intensiva. [Internet] 2010 [acesso em2010 fev]; 22(1): 85-91. Disponível em: http://rbti.org.br/rbti/download/artigo_2010512152044.pdf

12. Gerovasili V, Stefanidis K, Vitzilaios K, Karatzanos E, Politis P, Koroneos A, et al. Electrical muscle stimulation preserves the muscle mass of critically ill patients. A randomized study. Critical Care. [Internet] 2009 [acessoem2009 out]; 13(5): R161. Disponívelem: http://www.ncbi.nlm.nih.gov/pubmed/19814793

13. Zanotti E, Felicetti G, Maini M, Fracchia C. Peripheral muscle strength training in bed-bound patients with COPD receiving mechanical ventilation: effect of electrical stimulation. Chest. [Internet] 2003 [acesso em2003 jul]; 124(1): 292-6. Disponível em: http://www.ncbi.nlm.nih.gov/pubmed/12853536

14. Routsi C, Gerovasili V, Vasileiadis I, Karatzanos E, Pitsolis T, Tripodaki E, et. al. Electrical muscle stimulation prevents critical illness polyneuromyopathy: a randomized parallel intervention trial. CriticalCare. [Internet] 2010 [acesso em2010 abr 28]; 14(2): R74. Disponível em: http://www.ncbi.nlm.nih.gov/pubmed/20426834

15. Deley G, Kervio G, Verges B, Hanneguin A, Oetutdabt MF, Salmi-Belmihoub S, et. al. Comparison of low-frequency electrical myostimulation and conventional aerobic exercise training in patients with chronic heart failure. Eur J CardiovascPrevRehabil. [Internet] 2005 
[acesso em2005 jun]; 12(3): 226-33.Disponível em:http://www.ncbi.nlm.nih.gov/pubmed/15942420

16. Nuhr MJ, PetteD, Berger R, QuittanM, CrevennaR, Wiesinger GF, et al. Beneficial effects of chronic low-frequency stimulation of thigh muscles in patients with advanced chronic heart failure. Eur Heart J. [Internet] 2004 [acesso em2004 jan]; 25(2): 136-43. Disponível em:http://www.ncbi.nlm.nih.gov/pubmed/14720530

17. Bouletreau P, Patricot MC, Saudin F, Guiraud M, Mathian B. Effects of intermittent electrical stimulations on muscle catabolism in intensive care patients. J Parenter Enteral Nutr. [Internet] 1987 [acesso em1987 nov-dez]; 11(6): 552-5. Disponível em: http://www.ncbi.nlm.nih.gov/pubmed/3501482

18. Plank LD, Connolly AB,Hill GL. Sequential changes in the metabolic response in severely septic patients during the first 23 days after the onset of peritonitis. Ann Surg. [Internet] 1998 [acessoem1998 ago]; 228(2): 146-158. Disponível em: www.ncbi.nlm.nih.gov/pmc/articles/PMC1191454/

19. Eikermann M, Koch G, Gerwig M, Ochterbeck C, Beiderlinden M, Koeppen S, et. al. Muscle force and fatigue in patients with sepsis and multiorgan failure. IntensiveCareMed. [Internet] 2006 [acesso em2006 jan]; 32(2): 251-9. Disponível em:http://www.ncbi.nlm.nih.gov/pubmed/16468072

20. Gruther W, Benesch T, Zorn C, Paternostro-Sluga T, Quittan M, Fialka-Moser V, et. al. Muscle wasting in intensive care patients: ultrasound observation of the m. quadriceps femoris muscle layer. J Rehabil Med. [Internet] 2008 [acesso em2008 mar]; 40(3): 185-9. Disponível em: http://www.ncbi.nlm.nih.gov/pubmed/18292919

21. Ostrowski K, Rohde T, Zacho M, Pedersen BK. Evidence that interleukin-6 is produced in human skeletal muscle during prolonged running.J Physiol. [Internet] 1998 [acesso em 1998 mai]; 508(pt3): 949-953. Disponível em: http://www.ncbi.nlm.nih.gov/pmc/articles/PMC2230908/

22. Hirose L, Nosaka K, Newton M, Laveder A, Kano M, Peake J, Suzuki K. Changes in inflammatory mediators following eccentric exercise of the elbow flexors.ExercImmunol. [Internet] 2004 [acesso em2004]; 10: 75-90. Disponível em: http://www.ncbi.nlm.nih.gov/pubmed/15633588

23. Gerovasili V, Tripodaki E, Karatzanos E, Pitsolis T, Markaki V, Zervakis D, et al. Short term systemic effect of electrical muscle stimulation in critically ill patients. Chest. [Internet] 2009 [acessoem2009 ago 26]; 136(5): 1249-56. Disponível em: http://www.ncbi.nlm.nih.gov/pubmed/19710290

24. Oliveira Neto L, Sousa ADCV, Araújo AC. Idosos e treinamento de força: um estudo de revisão a partir da sarcopenia. EFDeportes, Revista Digital [Internet]. 2011 [acesso em2011 Jun]. Disponível em: http://www.efdeportes.com/efd157/idosos-e-treinamento-deforca-sarcopenia.htm 
25. Decramer M, De Benedetto D, Del Ponte A, Marianri S. Systemic effects of COPD. Respir Med. 2005; 99:S3-S10 Disponível em http://www.resmedjournal.com/article/S0954$6111 \% 2805 \% 2900368-9 /$ fulltext

26. Boschetti G. ¿Quées la estimulación? Teoría, práctica y metodología del entrenamiento. 2nd ed. Barcelona: Paidotribo; 2004.

27. DelittoAM, Brow MJ, Strube S, Rose J, Lehman RC. Electrical stimulation of quadriceps femoris in an elite weight lifter: a single subject experiment. Int J Sports Med. 1989; 10(3): 187-191 http://www.ncbi.nlm.nih.gov/pubmed/2674035

28. Brasileiro JS,Salvini TF. Limites da estimulação elétrica neuromuscular no fortalecimento de músculos esqueléticos saudáveis e com déficit de força. Fisioterapia Brasil. 2004; 5: 224-230. http://bases.bireme.br/cgi-bin/wxislind.exe/iah/online/

29. Matherson GP. Force output and energy metabolism during neuromuscular electrical stimulation: A 31 P-NMR study. Scand. J. Rehab. Med. 1997; 29: 17580.http://www.ncbi.nlm.nih.gov/pubmed/9271152

30. Guirro ECO, Guirro RRJ. Fisioterapia Dermato Funcional - Fundamentos, recursos e patologias. $3^{\text {a }}$ ed. São Paulo: Manole; 2002.

Figura 1 - Artigos encontrados, selecionados nas bases de dados aplicando os descritores estimulação elétrica, unidade de terapia intensiva e doente terminal.

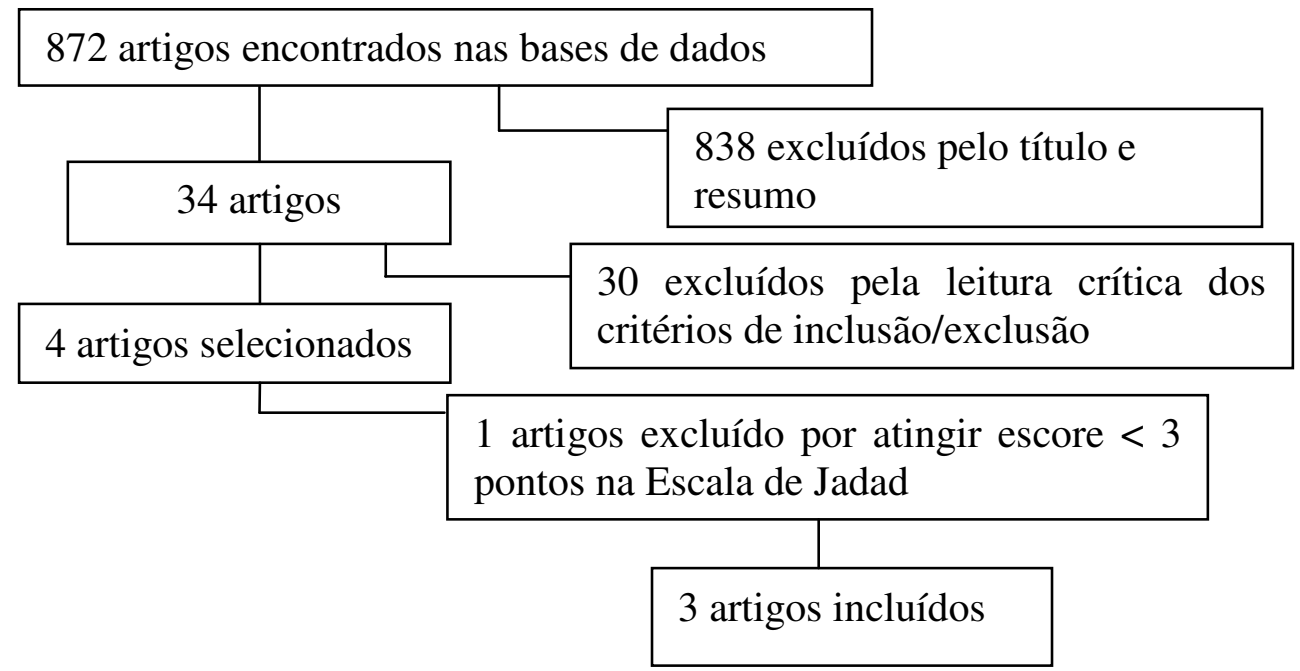


Tabela 1 - Análise Metodológica dos Ensaios Clínicos Randomizados incluídos na Revisão Sistemática Eletroestimulação em Doentes Críticos de acordo com aEscala JADAD, 2012.

\begin{tabular}{l|c|c|c}
\hline & Gruther et. al. $^{3}$ & Routsi et. al. $^{14}$ & Gerovasili et. al. $^{13}$ \\
\hline Alocação aleatória & Sim & Sim & Sim \\
\hline $\begin{array}{l}\text { Método de alocação } \\
\text { adequado }\end{array}$ & Sim & Sim & \\
\hline $\begin{array}{l}\text { Mascaramento } \\
\text { "duplo-cego" }\end{array}$ & Sim & Não & Não \\
\hline $\begin{array}{l}\text { Método de mascaramento } \\
\text { adequado }\end{array}$ & Sim & Não & Não \\
\hline $\begin{array}{l}\text { Descrição das perdas e } \\
\text { exclusões }\end{array}$ & Sim & Sim & Sim \\
\hline Escore total & 5 & 3 & 3 \\
\hline
\end{tabular}

Tabela 2 - Artigos incluídos na revisão sistemática Eletroestimulação em Doentes Críticos, 2012

\begin{tabular}{|c|c|c|c|c|}
\hline $\begin{array}{c}\text { Autor/Ano/ } \\
\text { Revista }\end{array}$ & $\begin{array}{c}\text { Características da } \\
\text { amostra }\end{array}$ & Objetivo & Intervenção & Efeitos encontrados \\
\hline $\begin{array}{l}\text { Gerovasili et. al., } \\
2009^{13} \\
\text { CriticalCare }\end{array}$ & 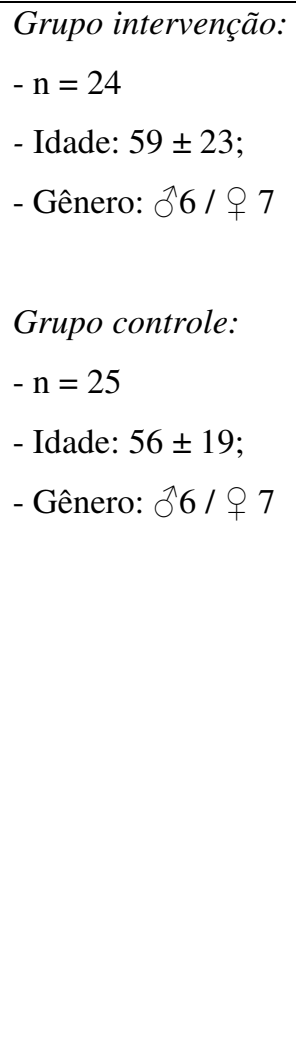 & $\begin{array}{l}\text { Avaliar o efeito } \\
\text { da EENM na } \\
\text { preservação da } \\
\text { massa muscular }\end{array}$ & $\begin{array}{l}\text { Grupo intervenção: EE } \\
\text { no quadríceps e fibular } \\
\text { longo de ambos os } \\
\text { MMII, aplicados do } 2^{\circ} \\
\text { ao } 9^{\circ} \text { dia de internação } \\
\text { (sessões de } 55 \text { min). } \\
\text { Grupo controle: Não } \\
\text { foram realizadas } \\
\text { intervenções neste } \\
\text { grupo }\end{array}$ & 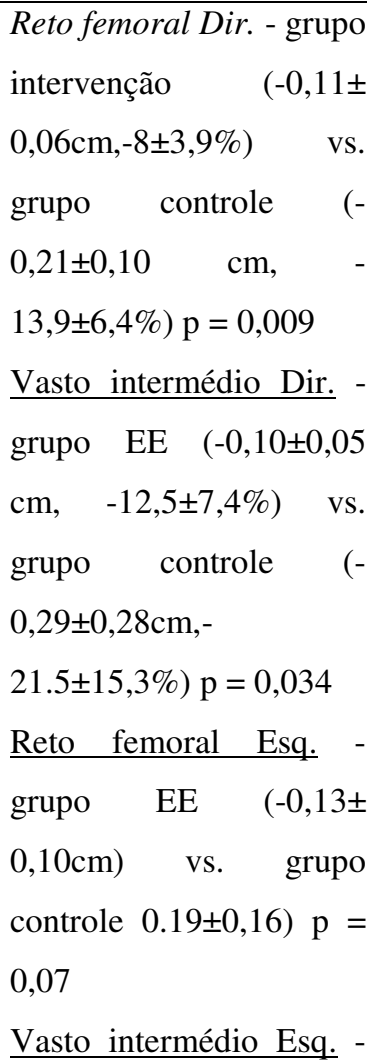 \\
\hline
\end{tabular}




\begin{tabular}{|c|c|c|c|c|}
\hline & & & & $\begin{array}{l}\left.\text { grupo } \begin{array}{l}\mathrm{EE}(-0,09 \pm 0,05 \\
\mathrm{cm} \pm\end{array} \quad 0,26\right) \mathrm{vs} \quad \text { grupo } \\
\text { controle } \quad(-0,22 \mathrm{~cm}) \quad \mathrm{p}= \\
0,018\end{array}$ \\
\hline $\begin{array}{l}\text { Routsi et. al., } \\
2010^{14} \\
\text { Critical Care }\end{array}$ & 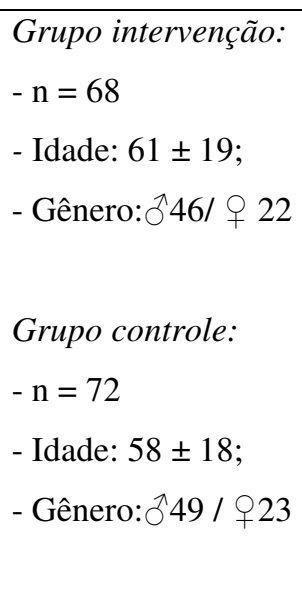 & $\begin{array}{l}\text { Avaliar a eficácia } \\
\text { da EENM na } \\
\text { prevenção da } \\
\text { polineuromiopatia } \\
\text { da doença crítica }\end{array}$ & $\begin{array}{l}\text { Grupo intervenção: EE } \\
\text { no vasto lateral, vasto } \\
\text { medial e fibular longo, } \\
\text { diariamente durante } 55 \\
\text { min até a alta da UTI. } \\
\text { Grupo controle: Não } \\
\text { foram realizadas } \\
\text { intervenções neste } \\
\text { grupo }\end{array}$ & $\begin{array}{l}\text { CIPNM: grupo EE }=3 \\
\text { pcts vs. grupo controle }= \\
11(\mathrm{p}=0,04) ; \\
\underline{\mathrm{n}^{\circ} \text { dias em VM: grupo EE }} \\
=4 \text { vs. grupo controle }= \\
6(\mathrm{p}=0,003) \\
\underline{\text { Tempo de internação: }} \mathrm{p} \\
=0,11\end{array}$ \\
\hline $\begin{array}{l}\text { Gruther et. al., } \\
2010^{3} \\
\text { Journal of } \\
\text { Rehabilitation } \\
\text { Med }\end{array}$ & 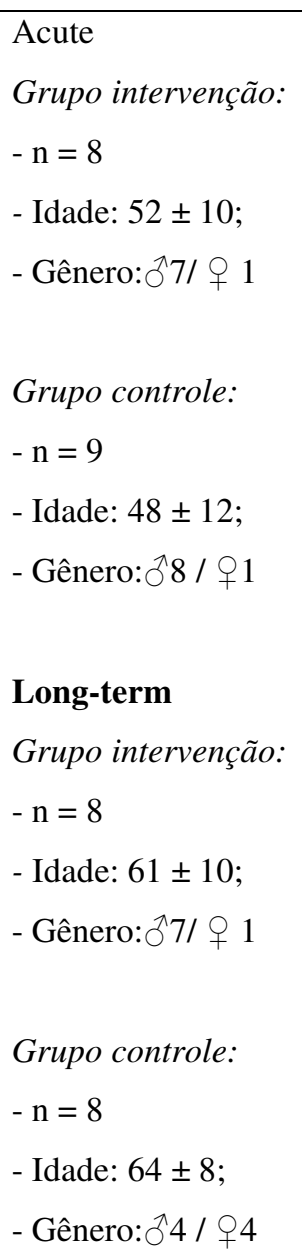 & $\begin{array}{l}\text { Avaliar a potência } \\
\text { da EENM em: (1) } \\
\text { retardar a perda } \\
\text { de massa } \\
\text { muscular quando } \\
\text { aplicado } \\
\text { precocemente, e } \\
\text { (2) reverter a } \\
\text { atrofia muscular } \\
\text { em pacientes a } \\
\text { longo prazo. }\end{array}$ & $\begin{array}{l}\text { Grupo intervenção: EE } \\
\text { no quadríceps, 1x por } \\
\text { dia, } 5 \mathrm{x} \text { por semana } \\
\text { durante } 4 \text { semanas. } \\
\text { Tempo de aplicação } 30 \\
\text { min/dia na primeira } \\
\text { semana, incrementado } \\
\text { para } 60 \text { min/dia a partir } \\
\text { da segunda semana. } \\
\text { Grupo controle: } \\
\text { Estimula a sensação de } \\
\text { formigamento, } \\
\text { ausência de contração } \\
\text { muscular. }\end{array}$ & $\begin{array}{l}\text { Redução da espessura da } \\
\text { camada } \\
\text { muscular:Paciente } \\
\text { agudos }(\mathrm{p}=0,45) ; \\
\text { Pacientes longo prazo (p } \\
=0,01)\end{array}$ \\
\hline
\end{tabular}


Tabela 3 - Parâmetros aplicados na eletroestimulação nos ensaios clínicos randomizados incluídos na Revisão Sistemática: Eletroestimulação em Doentes Críticos, 2012.

\begin{tabular}{lcccc}
\hline Artigo & Intensidade & Frequência & Duração do pulso & Ton/Toff \\
\hline Gerovasiliet al. $^{13}$ & Quadríceps: & $45 \mathrm{~Hz}$ & $400 \mu \mathrm{s}$ & $12 \mathrm{~s} / 6 \mathrm{~s}$ \\
& $\begin{array}{l}\text { 19-55mA } \\
\text { Fibular longo: } \\
23-60 \mathrm{~mA}\end{array}$ & & & \\
& - & $45 \mathrm{~Hz}$ & $400 \mu \mathrm{s}$ & $12 \mathrm{~s} / 6 \mathrm{~s}$ \\
Routsi et al. $^{14}$ & - & $50 \mathrm{~Hz}$ & $350 \mu \mathrm{s}$ & $8 \mathrm{~s} / 24 \mathrm{~s}$ \\
\hline
\end{tabular}

